\title{
Multiple Arthralgia After Use of Amoxicillin in EBV Infection: Could it be Adult Onset of Still's Disease?
}

\author{
Rachel WC Nga , Chi Eung Danforn Limª, ${ }^{\mathrm{a}}$, Nga Chong Lisa Cheng ${ }^{\mathrm{a}}$
}

\begin{abstract}
This case discusses about a rare development of adult-onset of still's disease in a 39-year-old male patient after taking amoxicillin for 14 days in an upper respiratory infection setting. It was confirmed later that he was in fact suffering from EBV infection at that time. Adult Onset Still's Disease (AOSD) is a rheumatic disease that tends to affect young adults between the ages of 16 and 35 . This article presents an uncommon case of AOSD after use of amoxillcin with EBV infection. Still's disease is thought to be triggered by external infectious agents in genetically predisposed hosts, with many clinical manifestations of AOSD similar to those of self-limited viral infections. Major clinical manifestations include transient fever, rash, arthralgia and arthiritis, myalgia and liver abnormalities. Diagnosis of AOSD is typically based on clinical observation. Treatment for AOSD is empirical and centers on the use of NSAIDs, steroids and disease modifying anti-rheumatic drugs.
\end{abstract}

Keywords: Athralgia; Antibiotics; Still's disease

\section{Introduction}

Adult Onset Still's Disease (AOSD) tends to affect young adults with $75 \%$ of reported cases of disease onset between the ages of 16 and 35 [1]. Due to its rarity, few large scale epidemiological studies have been conducted on AOSD, hence there is currently no global estimated incidence rate of the condition. However studies from various countries have been identified a yearly incidence rate of between

Manuscript accepted for publication January 14, 2014

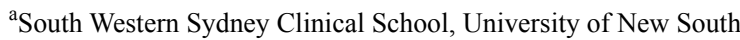
Wales, Australia

${ }^{\mathrm{b}}$ Corresponding author: Chi Eung Danforn Lim, PO Box 3256, Blakehurst, NSW 2221, Australia. Email: celim@unswalumni.com

doi: http://dx.doi.org/10.14740/jmc1661w
0.16/100,000 [2] and 0.4/100,000 [3]. An epidemiological study conducted in Japan reported an estimated crude prevalence rate of 0.73/100,000 in males and 1.47/100,000 in females [4].

\section{Case Report}

MC, a 39-year-old male, presented to local medical officer (LMO) with generalised body rash and polyarthropathy. MC had had recurrent episodes of tonsillitis four months prior to presentation, which was initially treated with amoxicillin for 2 weeks by another LMO. He then developed a diffuse pruritic erythematous rash across the abdomen which was thought to be an allergic reaction to amoxicillin, hence ceased. Roxithromycin and topical steroid cream was then prescribed to the $\mathrm{MC}$ resulting in resolution of pruritis, however rash and sore throat persisted for a total of 6 weeks. Serology results showed that MC was infected with Epstein-Barr virus and he was treated with amoxicillin at that time. EBV infection was also complicated by an erythematous rash and MC was given prednisone with effect. Subsequently, MC developed arthralgia involving both hands and knees and has since had intermittent episodes of arthralgia, rash, pyrexia, rigors and tonsillitis. LMO decided to refer the patient to hospital for urgent admission.

On presentation to hospital MC exhibited a low grade temperature of $37.6^{\circ} \mathrm{C}$ which spiked to $39.7^{\circ} \mathrm{C}$ on the ward. $\mathrm{He}$ also presented with tender PIP joints, swollen MCP joints and limited elbow flexion to 70 degrees. A diffuse erythematous macular rash was observed over limbs and abdomen, involving both flexor and extensor surfaces. Other findings included swollen lower limb and feet bilaterally, reduced capillary return in lower limbs and enlarged tonsils.

Laboratory tests returned the following results: 1), WCC 22 - $29 \times 10^{9} / \mathrm{L}$; 2), CRP $461 \mathrm{nmol} / \mathrm{L}$; 3), ESR $50-100 \mathrm{~mm} /$ $\mathrm{hr} ; 4)$, Ferritin peaked at $22,810 \mu \mathrm{g} / \mathrm{L}$.

During admission MC was thought to have Still's disease and was started on regular paracetamol and oxycontin, with PRN morphine, oxycodone and NSAID. Generalised arthralgia was not well controlled with oxycontin and PRN oxycodone/morphine, however pain was responsive to 
Table 1. Diagnostic Criteria of AOSD

\begin{tabular}{ll} 
Major Criteria & Minor Criteria \\
\hline Spiking fever $\geq 39^{\circ} \mathrm{C}$ & Maculopapular rash \\
Arthralgia & Leukocytes $\geq 10,000 / \mathrm{mm}^{3}$ \\
Transient erythema & \\
Pharyngitis & \\
PMN $\geq 80 \%$ & \\
Glycosylated ferritin $\leq 20 \%$ & \\
$* 4$ or more major criteria OR 3 major criteria +2 minor criteria
\end{tabular}

NSAIDs. Temperature spikes in early admission settled after increasing prednisone. Concurrent methotrexate was added to treatment regime with good tolerance and response of illness.

There was no further temperature spikes, joint swelling, improvement in range of movement and resolution of polyarthralgia following initiation of $75 \mathrm{mg}$ prednisone and 10 mg methotrexate.

\section{Discussion}

\section{Pathophysiology}

Like most rheumatic disease the pathophysiology of AOSD remains unclear. One hypothesis is that AOSD is a reactive syndrome triggered by external infectious agents in a genetically predisposed host. Viruses such as rubella, Epstein-Barr virus, mumps, adenovirus and parainfluenza all have been indicated [5]. The many clinical manifestations of AOSD that are similar to those of self-limited viral infections support this hypothesis.

\section{Clinical manifestations}

\section{Fever}

Fever which exceeds $39^{\circ} \mathrm{C}$ and transient in nature is typically seen in AOSD. It is usually quotidian or double quotidian in pattern with the highest temperature occurring the late afternoon or early evening period. The onset of fever usually precedes other manifestations such as sore throat, arthralgias, myalgias and serositis.

\section{Rash}

The classic rash seen in AOSD is usually described as an evanescent, salmon pink in color, maculopapular eruption and appears accompanying the fever spike. It is most frequently found on the proximal limbs and trunk and rarely on the distal limbs and face. It can be mildly pruritic which can be mistaken as a drug allergy.

\section{Arthralgia and arthritis}

Arthralgia and Arthritis occur in the majority of patients. Commonly affected joints include wrists, knees and ankles, however shoulder, elbow, distal and proximal interphalangeal joints, metatarsophalangeal and metacarpophalangeal joints, temporomandibular joints and hip have also been observed $[6,7]$. The arthritis pattern is generally symmetrical and most patients develop polyarthritis and joint pain associated with fever spikes.

\section{Myalgia}

Distribution of myalgia is generalized and often appears with fever exacerbations.

\section{Liver abnormalities}

Hepatomegaly and abnormal Liver function tests is also a common clinical manifestation but it cannot be excluded that non-steroidal anti-inflammatory drugs may contribute to this manifestation [5].

\section{Other}

Other less common manifestations include sore throat preceding fever and splenomegaly. Cardiopulmonary manifestations like tamponade, myocarditis, pleuritis and pericarditis have also been observed [5]. Hematological complications including pure red cell aplasia, hemolytic uremic syndrome and thrombotic thrombocytopenic purpura have also been 
noted [1].

\section{Diagnosis}

Diagnosis of AOSD is based on clinical observations. Several sets of diagnostic criteria have been published in regards to AOSD. Recently a new set of diagnostic criteria has been developed by Fautrel et al [8] incorporating the new finding of two diagnostic markers for AOSD (Table 1).

\section{Management}

Treatment for AOSD has remained exclusively empirical with treatment efficacy based largely on case reports and small scale retrospective studies. Treatment for AOSD largely centers on the use of NSAIDs, steroids and disease modifying anti-rheumatic drugs to control fever, arthritis and systemic disease. Other possible treatments include intravenous gammaglobulin and TNF blocking agents. However lack of large scale prospective, controlled treatment trials makes it difficult to validate any of these treatment regimens [5].

\section{Summary}

1), Use of Amoxicillin in setting of Tonsillitis should be avoided as it is difficult to differentiate viral or bacterial cause at the initial presentation of disease; 2), Onset of erythematous body rash after use of amoxicillin in URTI may not necessary related to allergic reaction as patient may have underlying EBV infection; 3), AOSD tends to affect young adults between the ages of 16 and 35; 4), Clinical manifestations of AOSD include fever spikes, rash, arthralgia and arthritis, myalgia and liver abnormalities; 5), Diagnosis is based on clinical observations with multiple major and minor criteria; 6), NSAIDs, steroids and disease modifying anti-rheumatic drugs have been used to treat AOSD, however multicentered, prospective, controlled treatment trials are needed to validate their effectiveness.

\section{Conflict of Interest}

None.

\section{References}

1. Bagnari V, Colina M, Ciancio G, Govoni M, Trotta F. Adult-onset Still's disease. Rheumatol Int. 2010;30(7):855-862.

2. Magadur-Joly G, Billaud E, Barrier JH, Pennec YL, Masson C, Renou P, Prost A. Epidemiology of adult Still's disease: estimate of the incidence by a retrospective study in west France. Ann Rheum Dis. 1995;54(7):587590.

3. Evensen KJ, Nossent HC. Epidemiology and outcome of adult-onset Still's disease in Northern Norway. Scand J Rheumatol. 2006;35(1):48-51.

4. Wakai K, Ohta A, Tamakoshi A, Ohno Y, Kawamura T, Aoki R, Kojima M, et al. Estimated prevalence and incidence of adult Still's disease: findings by a nationwide epidemiological survey in Japan. J Epidemiol. 1997;7(4):221-225.

5. Efthimiou P, Paik PK, Bielory L. Diagnosis and management of adult onset Still's disease. Ann Rheum Dis. 2006;65(5):564-572.

6. Pouchot J, Sampalis JS, Beaudet F, Carette S, Decary F, Salusinsky-Sternbach M, Hill RO, et al. Adult Still's disease: manifestations, disease course, and outcome in 62 patients. Medicine (Baltimore). 1991;70(2):118-136.

7. Masson C, Le Loet X, Liote F, Renou P, Dubost JJ, Boissier MC, Brithmer L, et al. Adult Still's disease: part I. Manifestations and complications in sixty-five cases in France. Rev Rhum Engl Ed. 1995;62(11):748-757.

8. Fautrel B, Zing E, Golmard JL, Le Moel G, Bissery A, Rioux C, Rozenberg S, et al. Proposal for a new set of classification criteria for adult-onset still disease. Medicine (Baltimore). 2002;81(3):194-200. 